\title{
A MOBILE BASED MICROSCOPE FOR SAMPLE RECOGNITION
}

\author{
Sagar K. Soni ${ }^{1}$, Pratik V. Patel ${ }^{2}$ \\ ${ }^{1}$ Student, Department of Information Technology, Dharmsinh Desai University, Nadiad, Gujarat, India \\ ${ }^{2}$ Research scholar, Research and Development Center, Dharmsinh Desai University, Nadiad, Gujarat, India
}

\begin{abstract}
Mobile phones provide important services like GPS, short-range wireless communications using infrared or Bluetooth, business applications, mobile banking etc. Mobile hard-ware add--ons are also popular like, handheld keyboard with mobile phone, mobile with handheld miniature microscope (phonoscope) and others. Imaging is one of the most important features of mobile technology. Magnified images are providing in depth recognition capabilities of object. Mobile based microscope is used for Geology, Biological, Medicine, Horticulture and others areas. Objective of this paper is to present a method for rock identification using mobile based microscope camera imaging of surface parameter. Rock surface parameters are color, grain, texture. The development in the area of mobile application has opened new challenges in mobile image processing. In this paper we demonstrate a method that adopts microscope optics into mobile camera optics and developed java based (J2ME) mobile application for recognizing rock type. This consists of feature extraction algorithm using wavelet based data compression and neural network based feature classification. Rock surface parameters used in this work is grain. The signature extracted from grain parameter is used to identify the rock type.
\end{abstract}

Keywords: Mobile image processing, Grain, Mobile microscope, Wavelet based data compression, Feature extraction, Neural net based classification.

\section{INTRODUCTION}

Rocks are an important category of Astor-materials. Rocks are the major composition of most planets like earth, moon and mars. The rocks are indication of evolution of a planet and its composition. Analysis and interpretation of earth rocks are mostly applicable to the rocks of other planets and meteorites.

Analysis of other planet rock samples has greatly helped in understanding of its mineral, chemical composition and age of associate important events. Rocks can transform from one type into another, as described by the geological model called the rock cycle. Geologist classifies rocks into three basic groups based on how they were formed in nature. Three general classes of rock: Igneous, Sedimentary and metamorphic.

Nowadays, the vast number of mobile phones that are available to the public offers a multitude of designs and functionalities. Mobile gives freedom and independence to communicate. Mobiles used vastly because of some reasons like portability, flexibility etc. Image processing on mobile phones is a new and very exciting field with many challenges due to limited hardware and connectivity.

Phones with Cameras, powerful CPUs, and memory storage devices are becoming increasingly common. Mobile applications face some challenges, because image processing operations can be very processor-intensive, especially if the image resolution is high. When combined with limited space for performing operations, mobile image processing tends to require small components that work with optimized algorithms.

We proposed to measure grain parameter of each rock surface and signature extracted from grain parameter is used to recognize rock type. The grain parameter is acquired using imaging sensors at different magnifications with handheld miniature microscope attached to a mobile phone. Handheld miniature microscope provide $60 \mathrm{x}-100 \mathrm{x}$ magnification. Here images taken from mobile microscope is transferred to javabased desktop applet application system which consists feature extraction algorithm using wavelet based data compression and neural net based feature classification and neural net training will be performed on desktop device which has more memory and resources. The generated neural net file is transferred to java based mobile application and testing of neural network is performed on mobile devices for identifying rock type. Specification of handheld miniature mobile microscope is as shown below table 1 
Tabel-1: Specification for Handheld Miniature Microscope

\begin{tabular}{|l|l|}
\hline Microscope & Universal handheld Miniature Microscope \\
\hline Magnification & $60 \mathrm{X}-100 \mathrm{X}$ magnification \\
\hline LED & $\begin{array}{l}\text { Inbuilt LED light with handheld Miniature } \\
\text { Microscope }\end{array}$ \\
\cline { 2 - 2 } & Power Supply: $3 \mathrm{x}$ LR1130 batteries \\
\hline Compatible & All mobile phone \\
\hline Application & $\begin{array}{l}\text { Geology, Biological, Medicine, Ore \& Jewellery, } \\
\text { Plate-making, Textile and other areas }\end{array}$ \\
\hline
\end{tabular}

Java Mobile Edition is a Java platform designed for embedded systems. The Connected limited device configuration (CLDC) is a specification of a framework for java mobile applications and it describing the basic set of libraries and virtual-machine features that must be present in an implementation. The Mobile Information Device Profile (MIDP) is a specification for the use of Java on embedded devices such as mobile phones. MIDP provide a GUI and a data storage API for java based application.

Here we demonstrate rock recognition system with testing neural net generated data file using java based mobile phone Samsung S5620 Monte device with CLDC 1.1 and MIDP 2.0 Version with camera captured on.

Tabel-2: Specification for Samsung S5620 Monte mobile phone

\begin{tabular}{|l|l|l|}
\hline \multirow{2}{*}{ Mobile Name } & \multicolumn{2}{|l|}{ Samsung S5620 Monte } \\
\hline \multirow{2}{*}{ Display } & Type & $\begin{array}{l}\text { TFT capacitive touch screen, } 256 \mathrm{~K} \\
\text { colors }\end{array}$ \\
\cline { 2 - 3 } & Size & $\begin{array}{l}240 \times 400 \text { pixels, 3.0 inches } \\
(\sim 155 \text { ppi pixel density })\end{array}$ \\
\hline Memory & Internal 200MB Storage \\
\hline $\begin{array}{l}\text { Operating } \\
\text { System }\end{array}$ & Samsung Proprietary OS, S40 Series \\
\hline Camera & $3.15 \mathrm{MP}, 2048 \times 1536$ pixels \\
\hline $\begin{array}{l}\text { Java } \\
\text { Supported }\end{array}$ & \multicolumn{2}{|l|}{ Yes, MIDP 2.0} \\
\hline
\end{tabular}

\section{PROPOSED ARCHITECTURE}

Here we proposed architecture for rock sample recognition.

\subsection{Proposed Architecture}

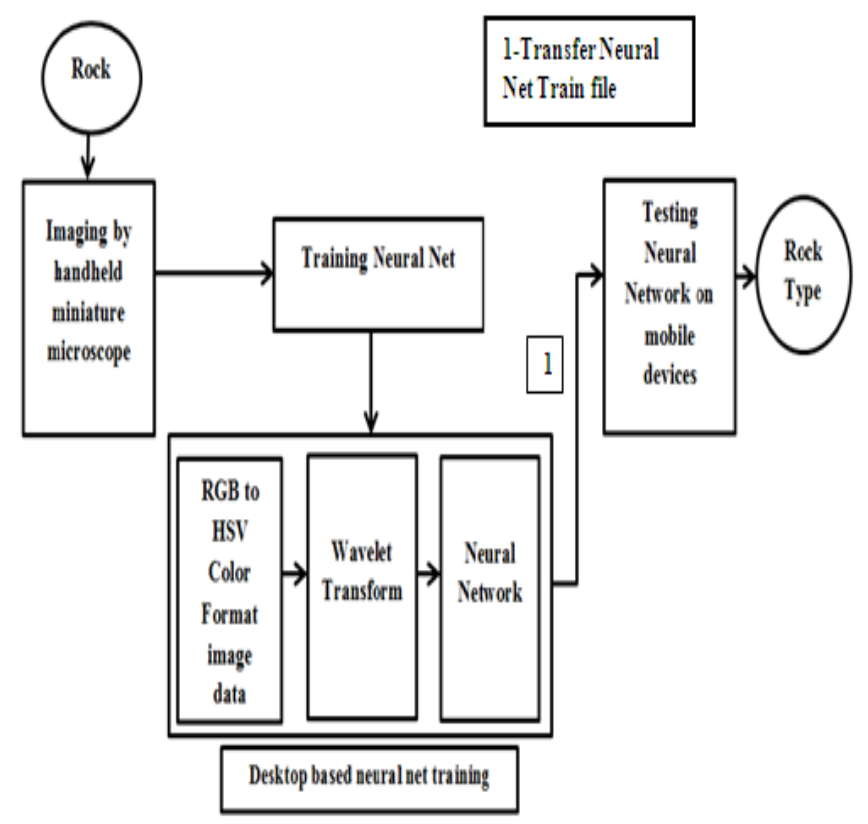

Fig-1 Block diagram of Proposed Architecture

Data Generator: In data generation take magnified grain images of rock surface with handheld mobile microscope and it transfer to java-applet based desktop system which consist feature extraction algorithm and neural net based rock image classification. Here $100 \mathrm{X}$ magnifications are used take magnified rock grain images.

Here system process is divided into two parts.

1) Training Neural Net part (Java-applet based desktop application)

2) Application part (Testing Neural net-on mobile devices)

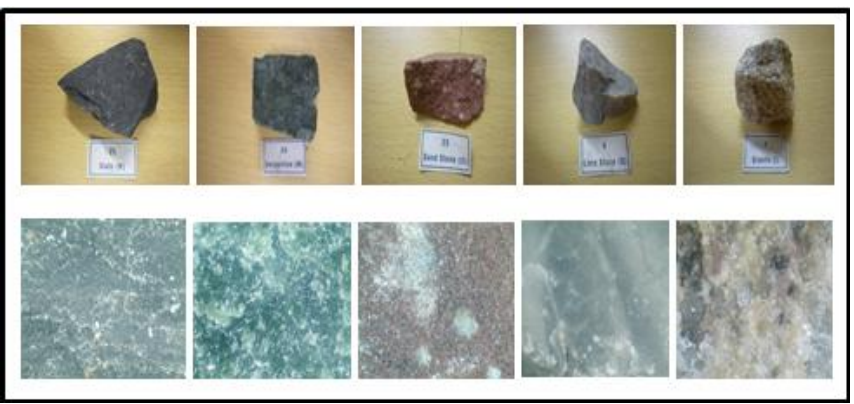

Fig-2 Top row shows five selected earth rock samples with corresponding magnified grain images with handheld mobile microscope 


\subsection{Training Neural Net}

This phase consist feature extraction algorithm using wavelet based data compression and neural network based feature classification techniques.

Convert RGB to HSV Color Format image data: Get RedGreen-Blue level data of rock grain images. HSV mathematical formula is used to convert RGB to HSV color format image data. George Paschos [1] compared and shown in his work that HSV color space performs better than $\mathrm{L} * \mathrm{a} * \mathrm{~b}$ and RGB.

To extract the color parameter independent of external illumination, the RGB space is converted to Hue, Saturation and intensity Value (HSV) parameters, where Hue represents the color component independent of color saturation and illumination intensity.

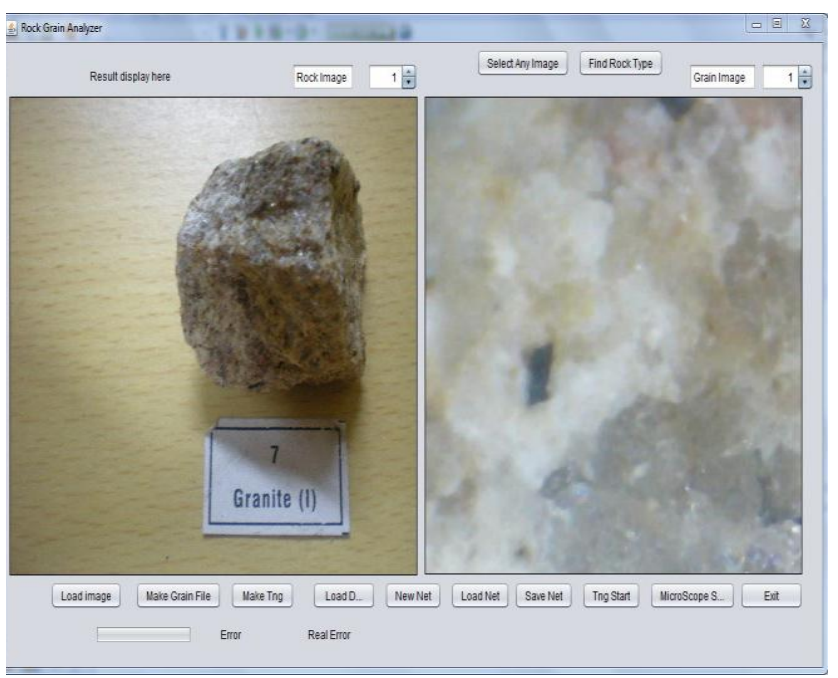

Fig-3 Screenshot of java-based applet application developed for Training neural net.

Hue ranges from $0^{\circ}$ to $359^{\circ}$ when measured in degrees. Saturation defines a range from pure color $(100 \%)$ to gray $(0 \%)$ at a constant lightness level and pure color is fully saturated. Lightness defines a range from dark $(0 \%)$ to fully illuminate $(100 \%)$. Any original hue has the average lightness level of $50 \%$.

Grain Analysis Algorithm: Rock grains refer to its mineral relationship with other nearest minerals. So it needs more magnified grain images of rock surface. To find nearest (close) rock minerals relationships from an entire rock grain image we design an algorithm that take two nearest random hue color-pair from a rock grain image and the most occurring hue color pairs are used for the grain analysis. Here 100x magnifications are used to capture rock grain images with mobile microscope.

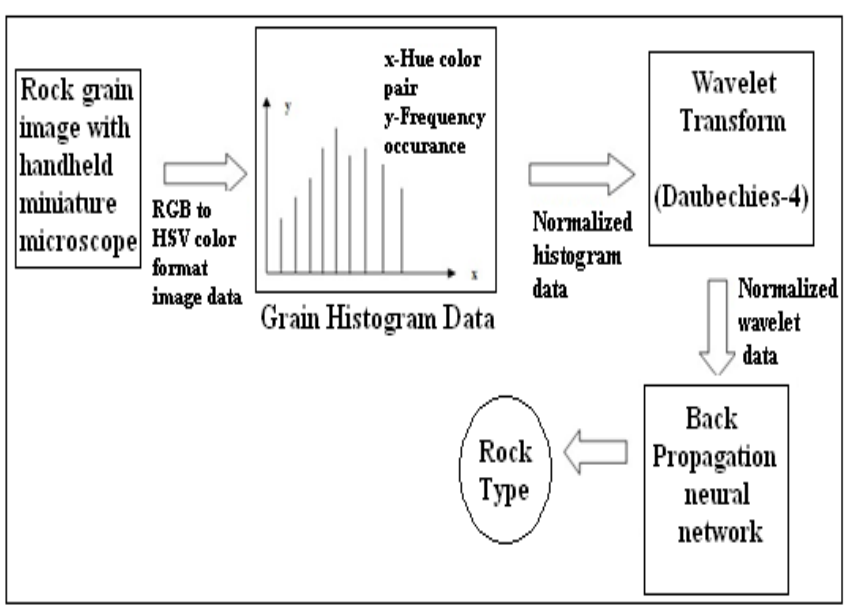

Fig-4 block-diagram of rock grain analysis algorithm

The hue color pair is selected randomly within range of 6 pixel (+/-3) distance. The distance 6 is selected to average $3 \times 3$ pixels area to represent grain. The sampling method used is color pair co-occurrence population.

Normalized grain feature values are applied to wavelet transform and output of wavelet transform is given as input in Neural Network back propagation training algorithm. All grain images used for this experiment are in JPEG format of resolution $1600 \mathrm{X} 1200$ in RGB color. Data is collected by sampling random pixels in the image area. Sampling methods for grain parameter is described in next section.

Sampling Method: For grain analysis each sample consists of a pair of pixels and the paired data is sampled between distances ranges of random $+/-3$. Total numbers of random samples used is in the range of 30,000 to $19,20,000$ depending on the accuracy and speed of recognition. These RGB samples are converted to HSV color format. In case of grain analysis only most occurring 235 pairs are used to restrict the input size. The hue pair value is used to calculate normalized histogram. The further processing consist of wavelet transform and neural network classification as described in section 2.3 and 2.4 below.

\subsection{Wavelet Transform}

Wavelets are used to convolve shape and texture with location information of image [2] [3]. Grain feature in our application are subjected to Daubechies wavelet [4]. The scaling and wavelet functions are calculated by taking the inner product of the coefficients and four data values. A segment of transformed values are selected and it used for classification. Different filter profile may be used to select appropriate segment of transformed values to optimize the accuracy and speed of classification. These selected transformed values are further processed using multilayer Neural Network. 


\subsection{Neural Network Architecture}

Wavelet transformed values are separately classified using multilayer Neural Network (NN) [5] with back-propagation training algorithm to identify rock type. Configuration of each $\mathrm{NN}$ used is three layer feed-forward type connections and consists of input, hidden and output layers. The NN grain parameter is uses 235 input neurons.

All inputs are normalized before applying to NN. All the three $\mathrm{NN}$ is configured using 10 hidden neurons and 5 output neurons. Each output corresponds to a rock type. Only one output is high at any time. Network is trained by computing the output error with respect to the desired output [6]. Numbers of hidden neurons are manually optimized for speed and accuracy.

The configuration of $\mathrm{NN}$ used for classification is shown in Figure 5. The Input, hidden and output layers are $\mathrm{Yi}, \mathrm{Yj}$ and Yk respectively. Desired output for training is dk. Xn and Yn are inputs and outputs of neurons. Output of NN is computed using equation 1, 2, 3 and 4 . Network is trained using error function given in equation 5. Error Sensitivity E/W of weights is calculated to reinforce the weights as shown in equation 6 and 7. Here two sigmoid activation functions are used. One is in hidden layer and other is in output layer.

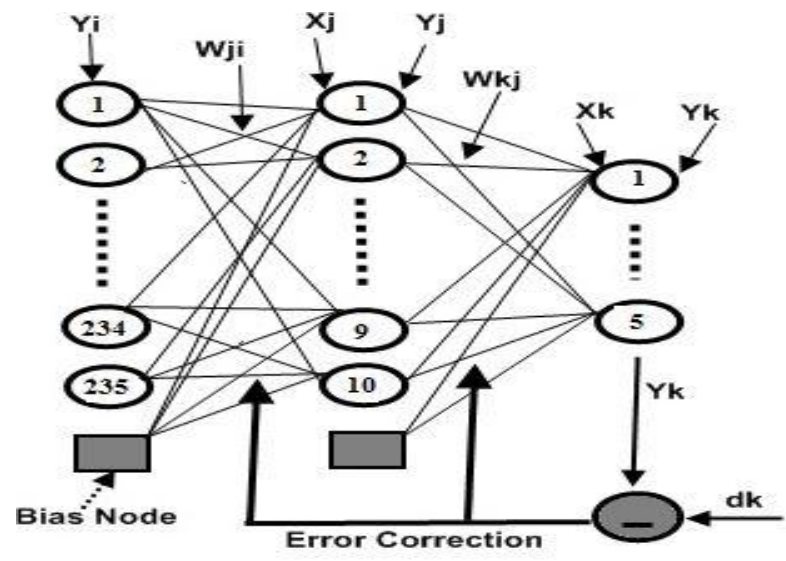

Fig-5 Shows a three layer Neural Network with Yi as inputs, Yk as outputs.

$$
\begin{aligned}
& \mathrm{Xj}=\Sigma \mathrm{i}=0 \mathrm{Yi} * \mathrm{Wji} \text { - } \mathrm{Yj}=1 /(1+\mathrm{e}-\mathrm{xj}) \\
& \mathrm{Xk}=\Sigma \mathrm{j}=0 \mathrm{Yj} * \mathrm{Wkj} \\
& \mathrm{Yk}=1 /(1+\mathrm{e}-\mathrm{xk}) \text { - } \\
& \mathrm{E}=0.5 *(\mathrm{Yk}-\mathrm{dk}) 2 \\
& \mathrm{Wkj}=\mathrm{Wkj}+\mathrm{eta} *(\mathrm{Yk}-\mathrm{dk}) * \mathrm{Yk} *(1-\mathrm{Yk}) * \mathrm{Yj}
\end{aligned}
$$

$$
\begin{array}{r}
\mathrm{Wji}=\mathrm{Wji}+\text { eta } * \mathrm{k}=0\{(\mathrm{Yk}-\mathrm{dk}) * \mathrm{Yk} \\
*(1-\mathrm{Yk})\} * \mathrm{Yj} *(1-\mathrm{Yj}) * \mathrm{Yi}--
\end{array}
$$

\section{EXPERIMENT SETUP}

In this Experiment setup we make an environment that suite handheld miniature mobile microscope and take fully focused image of object with constant distance, magnification and intensity for accurate sample recognition. In this section we demonstrate tools and rocks which are used in this experiment.

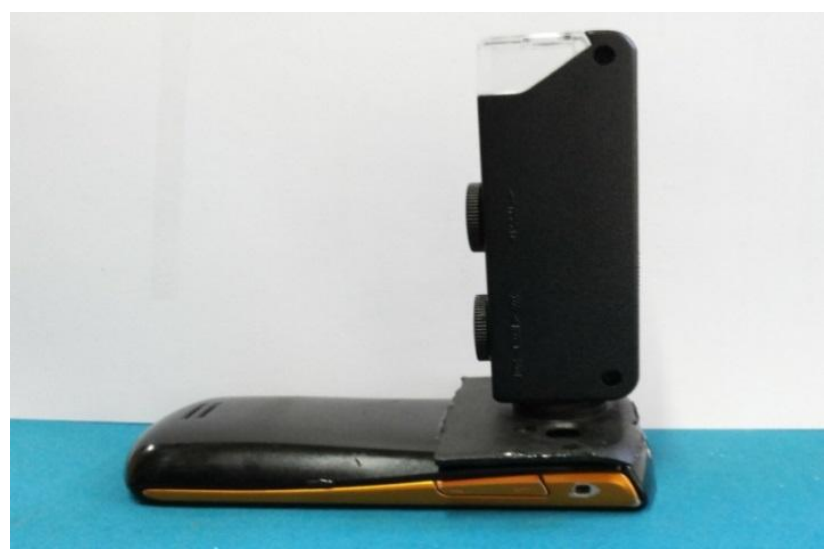

Fig-6 shows mobile with handheld miniature microscope

Auto adjustable hardboard with drilled four $6 \mathrm{~cm}$ nuts with mobile attached handheld microscope is shown below figure 7.

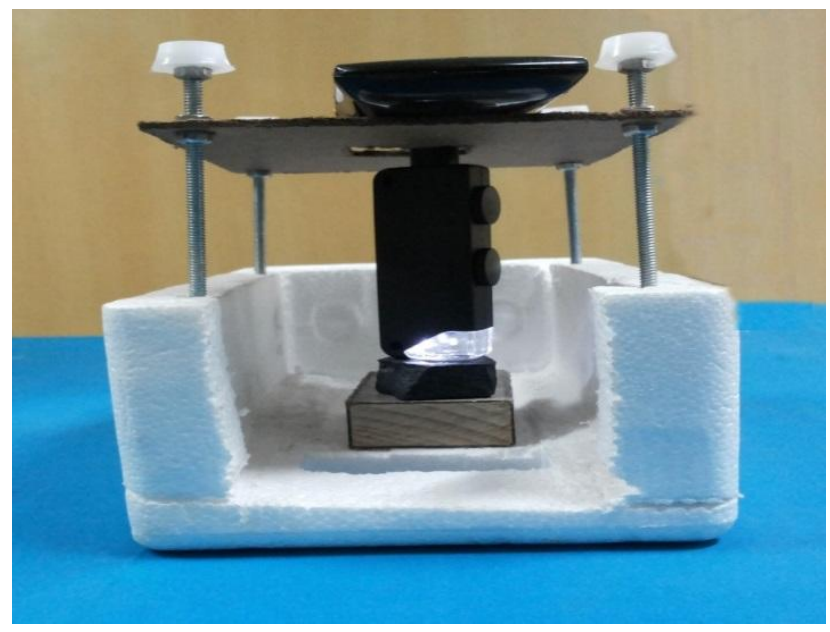

Fig-7 Environment setup for rock recognition

We take five different earth rocks Granite, Lime Stone, Sandstone, Serpentine, and Slate. These rocks are photographed using handheld miniature microscope attached with mobile camera. Rock images are shown in figure 2. Java based applet package trains the neural network and save neural net fie. Trained neural net file is transferred from desktop to mobile devices. Mobile application will configure neural net. 
Figure 8 shows rock grain image with mobile microscope and figure 9 shows mobile application.

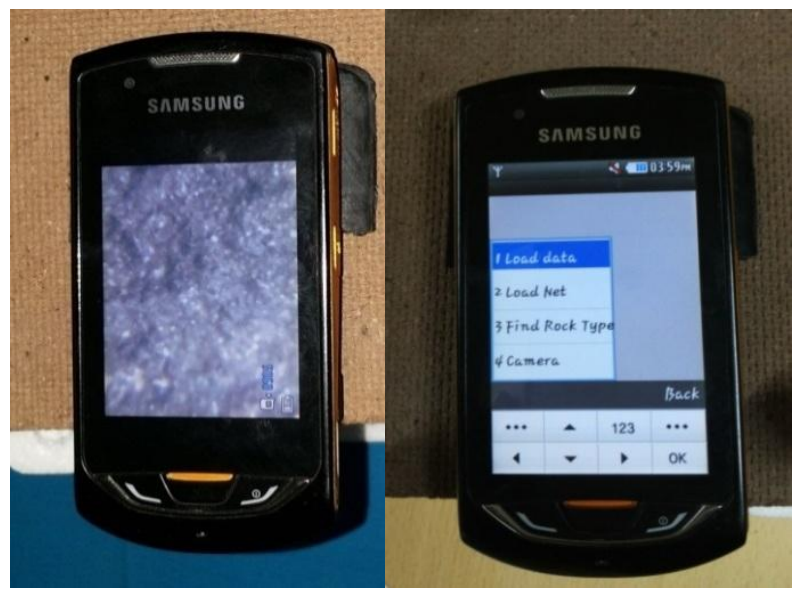

Fig-8 Display rock grain image Fig-9 shows J2ME Mobile with handheld miniature application for testing neural net Microscope

\section{EXPERIMENTAL RESULT}

Result presented here are from 5 rock types as described in section 3 and captured 50 grain images of rock with handheld miniature microscope. Number of training images are 40 and number of testing images are 10 for grain analysis. 10 images for each rock are taken and total 50 grain images are consider to evalute the algorithm performance.Table 3 shows result of rock grain analysis system.

Tabel-3: Result of rock grain analysis

\begin{tabular}{|c|c|c|c|c|}
\hline \multirow[t]{2}{*}{ Feature } & \multirow{2}{*}{$\begin{array}{l}\text { No. of } \\
\text { Training } \\
\text { images }\end{array}$} & \multirow{2}{*}{$\begin{array}{c}\text { No .of } \\
\text { Testing } \\
\text { images }\end{array}$} & \multicolumn{2}{|c|}{ Average accuracy } \\
\hline & & & $\begin{array}{l}\text { Untrained } \\
\text { test } \\
\text { samples } \\
(\%)\end{array}$ & $\begin{array}{l}\text { Trained } \\
\text { test } \\
\text { samples } \\
(\%)\end{array}$ \\
\hline $\begin{array}{l}\text { Rock } \\
\text { Grain }\end{array}$ & 40 & 10 & 90 & 95 \\
\hline
\end{tabular}

The output is shown in figure 10. Left figure shows output of rock named slate and Right figure shows rock named granite.
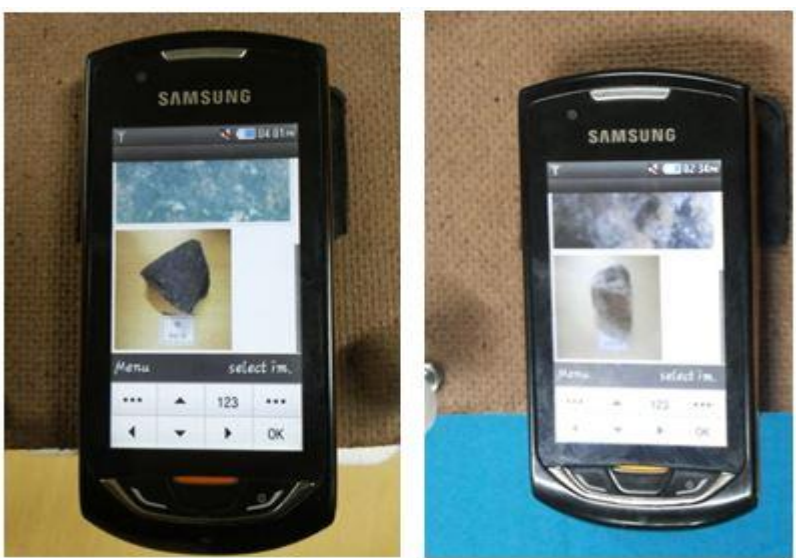

Fig-10 Output rock type of selected rock image

Testing on different mobile devices: Rock grain analysis mobile application is tested on various java based mobile devices like Nokia (E-66) s60 series, Sony Ericson (T-715) and Nokia E-77 with following specification

- $\quad$ CLDC $-1.1 \&$ MIDP - 2.0

- Phone should allow accessing Camera/Video by third party application and enable camera captured on.

\section{CONCLUSIONS}

New technology mobile based rock sample image identification/recognition is developed. Rock surface parameters are color, grain and texture. These are visual parameter of any rocks and it relate to rocks minerals composition. Microscopy images were taken using lab developed handheld miniature mobile microscope consist of Java based phone with appropriate lens attachments.

The single 100x image was used to identify rock grain. Pixel color histogram, wavelet transform of histogram and multilayer feed forward neural network is used to successfully analyzing and classifying earth rock with $95 \%$ of average accuracy for train samples and $90 \%$ of average accuracy of new untrained samples. Classification accuracy is further improved using multi-parameter analysis of different surface parameters. This method could be used in more application like horticulture, biological sample, precision stone identification, plate-making, textile, printed circuit boards and other industrial areas. In future android based application is also developed for different sample recognition.

\section{ACKNOWLEDGEMENTS}

While bringing out this paper to its final form, I came across a number of people whose contributions in various ways helped my field of research and they deserve special thanks. It is a pleasure to convey my gratitude to all of them. i would like to take this opportunity to express my gratitude and heartiest thanks to Dr. H. S. Mazumdar, head of department, Research and Development Center, DDU for his support and constant 
encouragement. Without his patient guidance, this work could never have been success. I convey my sincere thanks to the Head of the Department of the Information Technology Prof. R. S. Chhajed for kindly providing resources whichever needed for my dissertation.

\section{REFERENCES}

[1] Paschos, G."Perceptually uniform color spaces for color texture analysis: an empirical evaluation," Image Processing, IEEE Transactions on , vol.10, no.6, pp.932-937,Jun 2001doi: 10.1109/83.923289 K. Elissa, "Title of paper if known," unpublished.

[2] Dipankar Hazra, "Texture Recognition with combined GLCM, Wavelet and Rotated Wavelet Features". International Journal of Computer and Electrical Engineering, Vol.3, No.1, February, 2011

[3] Amara Graps "An Introduction to Wavelets", 1995 Institute of Electrical and Electronics Engineers. published by the IEEE Computer Society, 10662 Los Vaqueros Circle, Los Alamitos, CA 90720, USA,TEL +1-714-821-8380, FAX +1-714-821-4010.

[4] Dipankar Hazra, "Texture Recognition with combined GLCM, Wavelet and Rotated Wavelet Features". International Journal of Computer and Electrical Engineering, Vol.3, No.1, February, 2011

[5] Mehdi Lotfi, Ali Solimani, Aras Dargazany, Hooman Afzal, Mojtaba Bandarabadi, "Combining Wavelet Transforms and Neural Networks for Image Classification". 41st Southeastern Symposium on System Theory University of Tennessee Space Institute Tullahoma, TN, USA, March 15-17, 2009.

[6] Himanshu S. Mazumdar \& Leena P. Rawal, "A Neural Network Tool Box using C++ ", in CSI Communications, August, pp. 15-23, Bombay, 1995.

[7] DipankarHazra "Texture Recognition with combined GLCM, Wavelet and Rotated Wavelet Features"International Journal of Computer and Electrical Engineering, Vol.3, No.1, February, 2011

[8] TossapornKachanubal, and SomkaitUdomhunsakul "Rock Textures Classification Based on Texturaland Spectral Features".International Journal of Information and Mathematical Sciences.2008.

[9] LeenaLepisto, Iivari Kunttu1, JormaAutio, and Ari Visa "Classification Method forColored Natural Textures Using Gabor Filtering'International Conference on Image Analysis and Processing.

[10] Mehdi Lotfi1, Ali Solimani, Aras Dargazany, HoomanAfzal, MojtabaBandarabadi "Combining Wavelet Transforms and Neural Networks for ImageClassification"University of Tennessee Space Institute Tullahoma, TN, USA, March 15-17, 2009.

[11] SooBeom Park, Jae Won Lee, Sang KyoonKim"Content-based image classification using a neural network'.Pattern Recognition Letters 25 (2004) 287-300
[12] Himanshu S. Mazumdar \& Leena P. Rawal, "A Neural Network Tool Box using C++ ", in CSI Communications, August, pp. 15-23, Bombay, 1995

\section{BIOGRAPHIES}

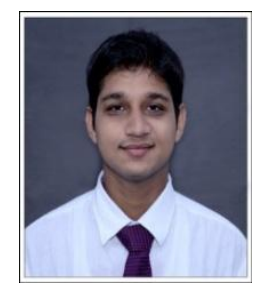

Sagar K. Soni received his Bachelor's degree in the year of 2012 and Master degree in in the year 2014 from Information Technology department. He has worked as Research scholar at R\&D Center of Dharmsinh Desai University, Nadiad, Gujarat, India.

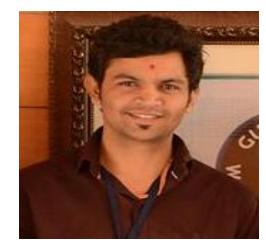

Pratik V. Patel received his Bachelor and Master degree of Computer Application in the year 2009 and 2011 respectively from Dharmsinh Desai University. $\mathrm{He}$ is working as Software engineer and Ph.D. research scholar at R\&D Center of Dharmsinh Desai University, Nadiad, Gujarat, India. 